\title{
Effects of freshwater clam extract on fracture induced inflammation at early stage
}

\author{
KUANG-TING YEH ${ }^{1,2}$, WEN-TIEN WU ${ }^{1,3}$, YI-MAUN SUBEQ ${ }^{4}$, CHI-CHIEN NIU ${ }^{5}$, \\ KUANG-WEN LIAO ${ }^{6}$, ING-HO CHEN ${ }^{2,3}$ and RU-PING LEE ${ }^{1}$ \\ ${ }^{1}$ Institute of Medical Sciences, Tzu Chi University, Hualien 97004; ${ }^{2}$ Department of Orthopedics, \\ Hualien Tzu Chi Hospital, Buddhist Tzu Chi Medical Foundation, Hualien 97002; ${ }^{3}$ School of Medicine and \\ ${ }^{4}$ Department of Nursing, Tzu Chi University, Hualien 97004; ${ }^{5}$ Department of Orthopaedic Surgery, \\ Chang Gung Memorial Hospital, Taoyuan 33305; ${ }^{6}$ Department of Biological Science and Technology, \\ National Chiao Tung University, Hsin-Chu 30010, Taiwan, R.O.C.
}

Received July 22, 2015; Accepted October 21, 2017

DOI: 10.3892/etm.2017.5184

\begin{abstract}
The inflammatory process after traumatic fracture and soft tissue injury includes release of inflammatory cytokines and activated polymorph nuclear cells (PMN) that can cause subsequent affected limbs delayed healing and vital organ complications. Analgesics have good effect on relief of the symptom but may cause further burden for hepatic and renal metabolism. Freshwater clam extract (FCE) has been demonstrated to suppress the release of the pro-inflammatory cytokine tumor necrosis factor- $\alpha$ production after hemorrhagic shock, and decrease the level of liver injury marker in rats. The aim of the present study was to determine whether FCE is able to affect the inflammation induced by unilateral tibial fracture in a rat model. The rats were randomly divided into control, fracture, FCE and fracture with FCE groups. The fracture group received left tibia and fibula shaft fractures using a consistent three point bending method. For the fracture with FCE group, FCE $(40 \mathrm{mg} / \mathrm{kg})$ was administered orally after fracture. Their physiological changes were continuously monitored for $48 \mathrm{~h}$. Blood samples were extracted from the femoral arterial catheter at 1, 3, 6, 9, 12, 18, 24 and $48 \mathrm{~h}$ after fracture. In comparison with fracture group, those whom were fed with FCE had more stable heart rate frequency, lower central temperature at the initial $\mathrm{h}$, and lower serum level of the proinflammatory cytokines and muscle damage markers induced by fracture. FCE was also associated with decreased recruitment of inflammatory cells in the adjacent soft tissue. Thus, the present results suggest that FCE could decrease
\end{abstract}

Correspondence to: Dr Ru-Ping Lee, Institute of Medical Sciences, Tzu Chi University, 701 Zhongyang Road, Sec. 3, Hualien 97004, Taiwan, R.O.C.

E-mail: fish@mail.tcu.edu.tw

Key words: proinflammatory cytokines, freshwater clam extract, conscious rat model, fracture fracture induced inflammation reaction and have beneficial regulatory effect on post inflammatory response.

\section{Introduction}

Trauma is a common reason for the admission of patients to an emergency room. Bone fracture combined with soft tissue contusion is the most common injury seen by an orthopedic doctor; local tenderness, pain and swelling are the most troublesome and frequent problems (1). More than $90 \%$ of multiply injured patients with bone fractures are related with the development of the systemic inflammatory response syndrome and significantly correlate with multiple organ failure, sepsis and mortality (1). The inflammatory process includes release of inflammatory cytokines, activated polymorph nuclear cells (PMN) and the expression of leukocyte adhesion molecules (2). Anti-inflammatory treatment may in certain cases result in delayed infection (3). Therefore, regulation of cytokine release following trauma has been considered a potential strategy (4), and may improve patients' life quality during acute disease stage. Various anti-inflammatory medication and analgesics may be selected, but may cause allergic reaction or more burden to the stomach, liver or kidney which may become injured during the acute stages of trauma (5).

Previous results have suggested that bone fracture elicited a distinct pattern of cytokines associated with organ injury (6). The data revealed similar patterns of interleukin-6 (IL-6) and IL-10 after isolated bilateral femur fracture as compared with other injury ways (6); however, tumor necrosis factor- $\alpha$ (TNF- $\alpha$ ) and IL-1 $\beta$ exhibit different patterns of a higher level maintained over a longer time period. A minor unbalance in serum cytokines or locally produced cytokines may be responsible for early stage PMN infiltration of the organ (2).

Macrophages and monocytes are the main producers of TNF- $\alpha$, that may stimulate the secretion of a variety of proand anti-inflammatory cytokines, for example IL-6, IL-8 and IL-10 (7). Either anti-TNF- $\alpha$ antibodies or soluble receptors for TNF- $\alpha$ have become a strategy in the treatment of patients with chronic inflammatory diseases through attenuating TNF- $\alpha$ (4). Overwhelming production of pro-inflammatory 


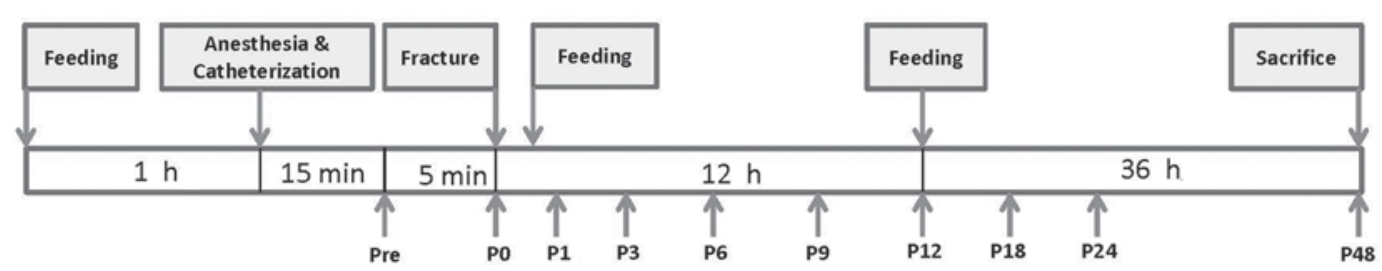

Figure 1. Flow chart of experimental design of this study.

cytokines such as TNF- $\alpha$ and uncontrolled production of anti-inflammatory cytokines such as IL-10 can lead to multiple organ dysfunction and mortality (4). Trauma, taking fracture as an example, induces inflammation that can cause elevation of proinflammatory cytokines and induce a series of systemic injury (1).

Freshwater clams (Corbicula fluminea), are a popular edible shellfish in Asia. Recent experiments noted that freshwater clam extract (FCE) exerts anti-oxidative and anti-inflammatory activity (8). FCE suppresses the release of the pro-inflammatory cytokine TNF- $\alpha$ production after hemorrhagic shock and decreased the level of liver injury marker in rats (9). Oral FCE also significantly decreased chronic hepatic fibrosis triggered by carbon tetrachloride through attenuating thiobarbituric acid reactive substances, hydroxyproline and excessive inflammation in rats (10). To the best of our knowledge, no prior studies have investigated the effects of FCE consumption on acute trauma conditions.

The aim of the present study was to determine whether FCE affected the inflammation induced by unilateral tibial fracture in a conscious rat model.

\section{Materials and methods}

Preparation of FCE and experimental animals. We prepared FCE and quantitated its anti-inflammation activity with previous method (11). A total of 32 male Wistar-Kyoto rats weighing 280-320 g were purchased from the National Animal Center (Taipei, Taiwan). The rats were housed in our animal center under a controlled environment at $22 \pm 1^{\circ} \mathrm{C}$ with a $12-\mathrm{h}$ light/dark cycle. Food and water were provided ad libitum. The experimental protocol was approved by the Animal Usage Regulation Committee of Tzu Chi Hospital (Hualien, China).

Conscious rat model of closed tibia fracture. The animals were anesthetized with ether inhalation for $10 \mathrm{~min}$. During the period of anesthesia, polyethylene catheters (PE-50) were inserted into the femoral artery for collecting blood samples. The operation was completed within $15 \mathrm{~min}$, and the section wound was as small as possible $\left(<0.5 \mathrm{~cm}^{2}\right)$ (9). Following the operation, the animal was placed in an individual cage (Shingshieying Instruments, Hualien, Taiwan). After the rats were placed properly, left tibial shaft fracture was performed with three points bending method smoothly (12). The fractured limb was fixed with an external wooden stick wrapped with elastic bandage, which imitated the clinical management of the situation.

Experimental design. The animals were randomly divided into four groups. The feeding time-points were prefracture $1 \mathrm{~h}$, and post-fracture 0.5 and $11.5 \mathrm{~h}$. We placed the fed substance in the stomach of the rats using a dosing syringe. The sham $(n=8)$ and fracture (Fx) groups ( $n=8$ per group) were fed with $1 \mathrm{ml}$ normal saline (NS) at the three time-points. The fracture with FCE $(\mathrm{Fx}+\mathrm{FC})$ and the non-fracture with $\mathrm{FCE}(\mathrm{NFx}+\mathrm{FC})$ groups were fed with FCE $(20 \mathrm{mg} / \mathrm{kg})$ at each of the three time-points. Fig. 1 displays a flow chart detailing the study protocol.

Heart rate $(H R)$ and central temperature $(C T)$ recordings. The arterial catheter of was connected to a pressure transducer (Gould Instrument Systems, Cleveland, OH, USA) for recording mean arterial pressure (MAP) and HR, using a polygraph recorder (Power Lab; ADInstruments, Mountain View, CA, USA). CT was estimated and recorded continuously through the anal thermometer placed in the rectum of the rats.

Blood sample analyses. Arterial blood samples were obtained for baseline values before fracture. Arterial blood samples $(0.5 \mathrm{ml})$ were collected before fracture and at 1, 3, 6, 9, 12, 24 and $48 \mathrm{~h}$ after fracture. Half of the serum was stored at $-20^{\circ} \mathrm{C}$ for cytokine analysis. The concentrations of TNF- $\alpha$ (DY510), IL-6 (DY506) and IL-10 (DY522) in the samples were measured separately using enzyme-linked immunosorbent assay (ELISA) kits (R\&D Systems, Minneapolis, MN, USA). All samples were stored at $-20^{\circ} \mathrm{C}$ prior to testing. The reagents, samples and working standards were brought to room temperature and prepared according to the manufacturer's directions. The ELISA was performed following the manufacturer's instructions. Each sample was run in duplicate and determined using an automated ELISA reader (Sunrise; Tecan, Grödingen, Austria) at $450 / 540 \mathrm{~nm}$. The other half of the serum was decanted and stored at $4^{\circ} \mathrm{C}$. Biochemical examinations were performed within $1 \mathrm{~h}$ after specimen collection. Serum levels of creatine phosphokinase (CPK) and lactate dehydrogenase (LDH) were measured using an auto analyzer (COBAS Integra C111; Roche Diagnostics, Basel, Switzerland).

Histological examination. Tibial muscles were harvested $48 \mathrm{~h}$ after fracture. These tissue specimens were fixed overnight in $4 \%$ buffered formaldehyde, processed by standard methods, and stained with hematoxylin and eosin. An observer participated in the pathological examination in a blind fashion to minimize subjectivity.

Data analysis. Data are expressed as the mean \pm standard error of the mean. The significance of differences in the measured values between groups was analyzed using one-way analysis of variance. The significance of the measured values within groups was analyzed with repeated measurements by t-test. 

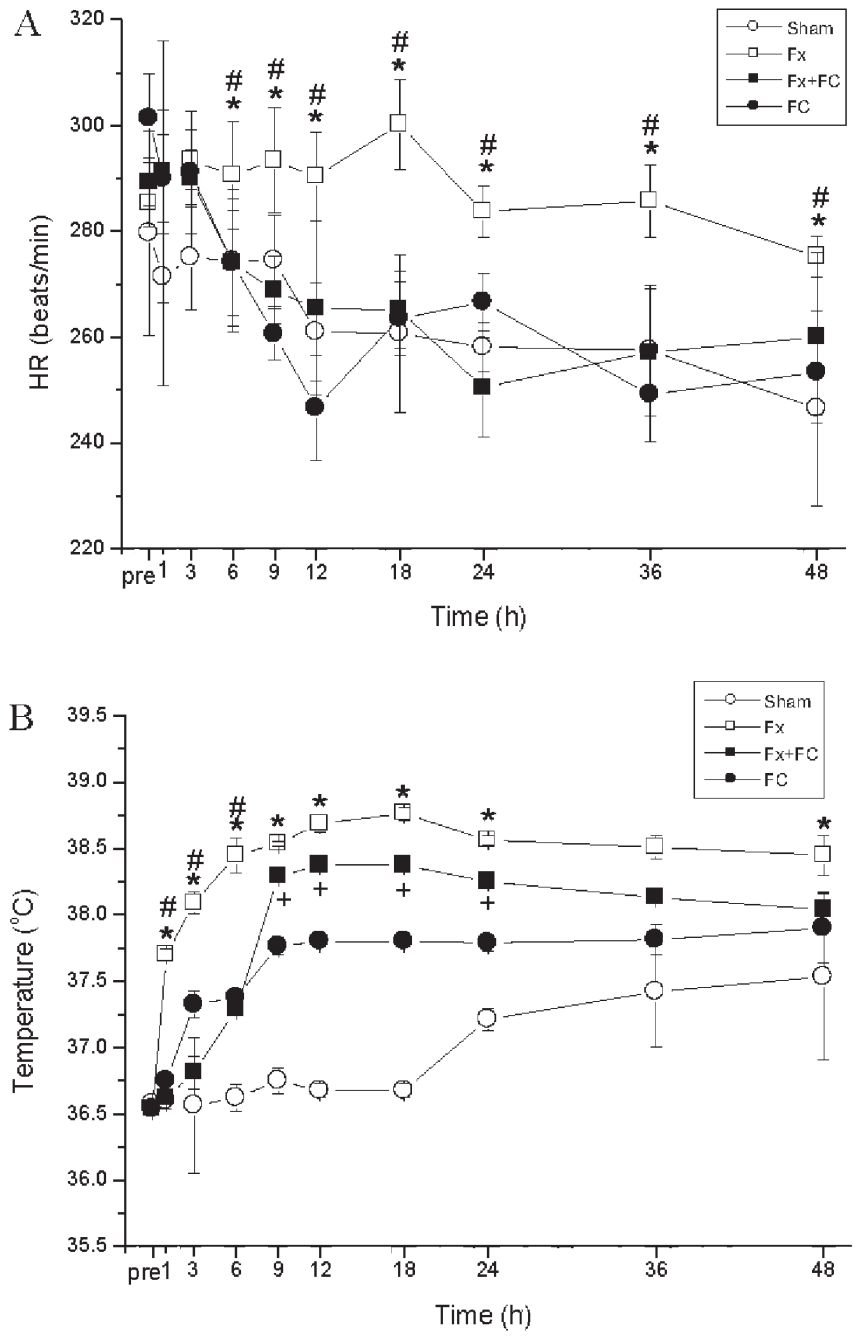

Figure 2. Time courses changes of (A) HR and (B) central temperature after fracture. Rats were separated into fracture $(\mathrm{Fx}$ and $\mathrm{Fx}+\mathrm{FC})$ and non-fracture (sham and FC) groups. Results are expressed as the mean \pm standard error. ${ }^{\#} \mathrm{P}<0.05$, Fx vs. Sham; ${ }^{*} \mathrm{P}<0.05$, Fx vs. Fx $+F C ;{ }^{+} \mathrm{P}<0.05, \mathrm{Fx}+\mathrm{FC}$ vs. Sham. HR, heart rate; $\mathrm{Fx}$, fracture group; $\mathrm{Fx}+\mathrm{FC}$, fracture and freshwater clam extract group.

$\mathrm{P}<0.05$ was considered to indicate a statistically significant difference.

\section{Results}

Effects of FCE on vital signs post inflammatory response. The average HR and CT showed no significant difference between four groups before blood withdrawal (Fig. 2). The Fx group had significantly higher HR levels at all the time-points compared to the sham group $(\mathrm{P}<0.05)$ (Fig. 2A). The $\mathrm{Fx}+\mathrm{FC}$ group had relatively lower HRs at 3, 6, 9, 12 and 24 h compared to the Fx group, although HR did not significantly change during the experimental period. CT of the Fx and sham groups decreased at $6 \mathrm{~h}$ and increased at $9 \mathrm{~h}$. The average CT of the Fx group was significantly increased at all the time-points compared to the baseline level $(\mathrm{P}<0.05)$ (Fig. 2B). CT of the Fx+FC group decreased at $1 \mathrm{~h}$ and increased steadily between 1 and $9 \mathrm{~h}$, and was unchanged between 9 and $24 \mathrm{~h}$. The CT of the Fx+FC group was significant lower at 1 and $3 \mathrm{~h}$, and higher at $6 \mathrm{~h}$, compared to the Fx group.
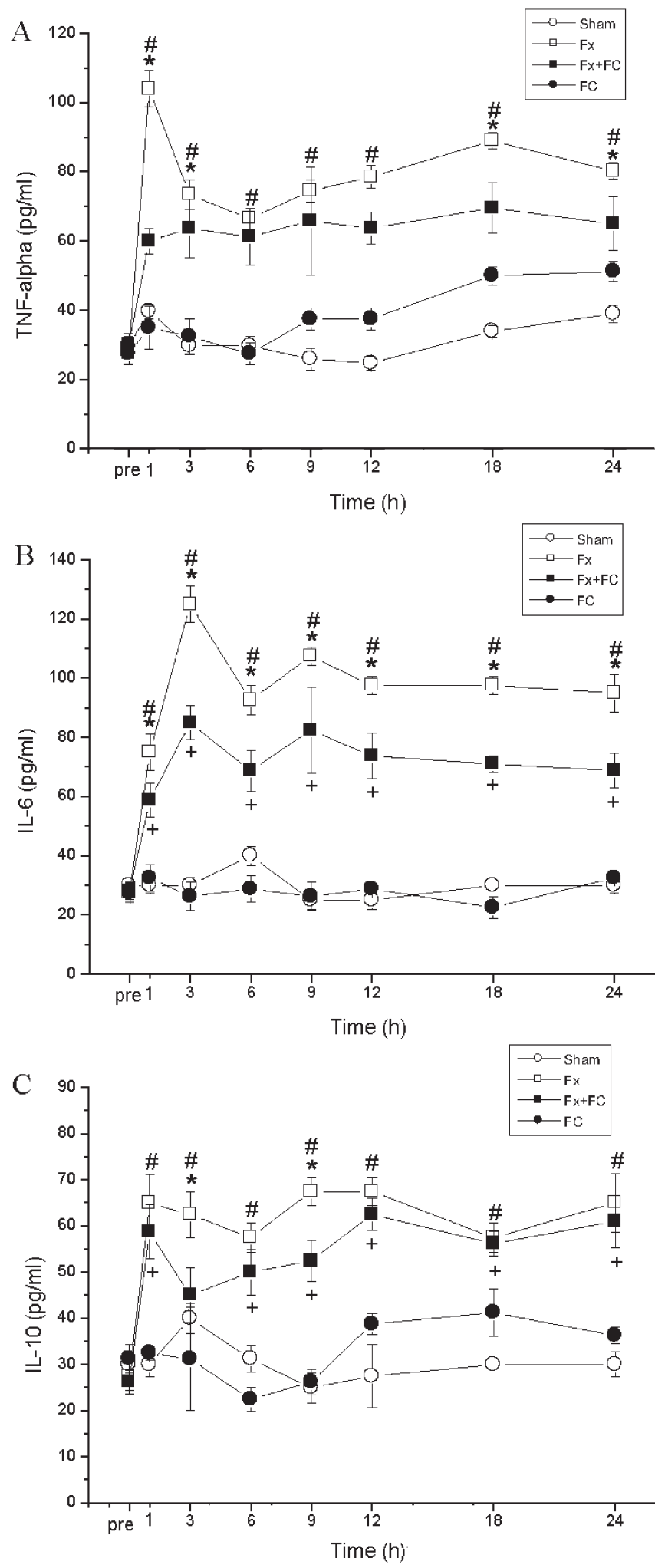

Figure 3. Changes of serum (A) TNF- $\alpha$, (B) IL-6 and (C) IL-10 level after tibia and fibula fracture. Results are expressed as the mean \pm standard error. ${ }^{\#} \mathrm{P}<0.05$, Fx vs. Sham; ${ }^{*} \mathrm{P}<0.05$, Fx vs. Fx+FC; ${ }^{+} \mathrm{P}<0.05, \mathrm{Fx}+\mathrm{FC}$ vs. Sham. $\mathrm{TNF}-\alpha$, tumor necrosis factor- $\alpha$; IL, interleukin; Fx, fracture group; Fx+FC, fracture and freshwater clam extract group.

Effects of FCE on proinflammatory cytokine serum level. Plasma levels of TNF- $\alpha$, IL- 6 and IL-10 showed no significant difference between the four groups prior to blood withdrawal (Fig. 3). 

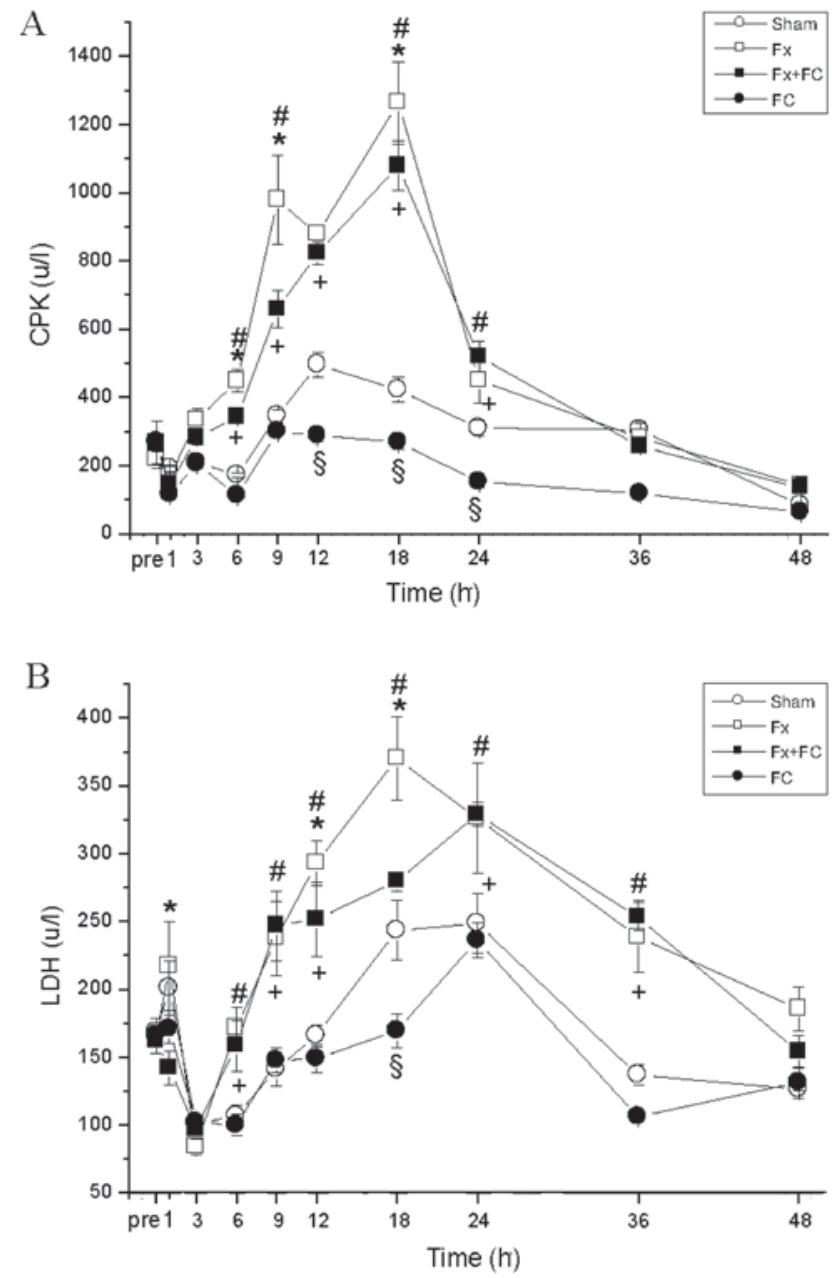

Figure 4. Changes of serum (A) CPK and (B) LDH level after tibia and fibula fracture. Results are expressed as the mean \pm standard error. ${ }^{\#} \mathrm{P}<0.05, \mathrm{Fx}$ vs. Sham; ${ }^{*} \mathrm{P}<0.05, \mathrm{Fx}$ vs. $\mathrm{Fx}+\mathrm{FC} ;{ }^{+} \mathrm{P}<0.05, \mathrm{Fx}+\mathrm{FC}$ vs. Sham; ${ }^{\circledR} \mathrm{P}<0.05, \mathrm{FC}$ vs. Sham. CPK, creatine phosphokinase; LDH, lactate dehydrogenase; Fx, fracture group; $\mathrm{Fx}+\mathrm{FC}$, fracture and freshwater clam extract group.

The plasma TNF- $\alpha$ level elevated to the first peak in the Fx group at $1 \mathrm{~h}$ and decreased rapidly at $3 \mathrm{~h}$ and mildly at $6 \mathrm{~h}$ $(\mathrm{P}<0.05)$ (Fig. 3A). Then it reached the second peak at $18 \mathrm{~h}$. TNF- $\alpha$ levels in the Fx group were significantly higher than that of the sham group $(\mathrm{P}<0.05)$. TNF- $\alpha$ levels of the Fx+FC group climbed up to the peak at $1 \mathrm{~h}$ and reduced to a steady level between 3 and $4 \mathrm{~h}$, which was significantly lower than that of the Fx group at 1,3, 9, 12, 18 and $24 \mathrm{~h}$.

Serum IL-6 levels of the Fx and Fx+FC groups were significantly higher compared to the sham group $(\mathrm{P}<0.05)$ (Fig. 3B). Serum IL-6 levels peaked at 3 and $9 \mathrm{~h}$ in the Fx group, and the former one is higher. The IL- 6 level of the Fx+FC group showed a similar pattern to the Fx group, but was significantly lower at all the time-points $(\mathrm{P}<0.05)$.

Serum levels of IL-10 were significantly higher in the Fx group compared with the sham group, and it had two reverse peak at 6 and $18 \mathrm{~h}$ after elevation at $1 \mathrm{~h}(\mathrm{P}<0.05)$ (Fig. 3C). The IL-10 level sof the Fx+FC group were significantly higher than the sham group at 1, 6, 9, 12, 18 and $24 \mathrm{~h}$, which had two reverse peaks at 3 and $18 \mathrm{~h}$ after elevation at $1 \mathrm{~h}$. IL-10 levels of the Fx+FC group were obviously reduced compared with the Fx group at 3 and $9 \mathrm{~h}$.
These results suggest that FCE lowered the levels of the proinflammatory cytokines that had been induced by fracture.

Effects of FCE on injury related enzyme post inflammatory response. Plasma CPK level reduced mildly at $1 \mathrm{~h}$, then increased in all four groups (Fig. 4A). The CPK levels of the $\mathrm{Fx}$ and $\mathrm{Fx}+\mathrm{FC}$ groups were significantly higher than the sham group at $3,6,9,12,18$ and $24 \mathrm{~h}(\mathrm{P}<0.05)$, and peaked at $18 \mathrm{~h}$. The CPK levels of the Fx+FC group were lower at 6, 9 and $18 \mathrm{~h}$ compared to the Fx group.

Plasma LDH levels of the Fx group peaked at 1 and $18 \mathrm{~h}$ (Fig. 4B). The LDH levels of the Fx and FC groups decreased at 1 and $3 \mathrm{~h}$, and peaked at $24 \mathrm{~h}$. The LDH levels in the Fx+FC group were significantly lower at 1, 12 and $18 \mathrm{~h}$, compared to the Fx group $(\mathrm{P}<0.05)$. LDH levels of the Fx and $\mathrm{FX}+\mathrm{FC}$ groups returned to prefracture level at $48 \mathrm{~h}$.

Effects of FCE on the recruitment of lymphocytes. Histopathological analysis of the hematoxylin and eosin-stained tissue sections was performed. There were no significant changes over the muscle in the sham group (Fig. 5A); however, this analysis revealed lymphocyte infiltration and hemorrhage of the muscle in the Fx group (Fig. 5B). Less grouping of lymphocytes and hemorrhage was noted in the Fx+FC group (Fig. 5C). There were no significant changes over the muscle in the FC group (Fig. 5D). These findings suggest that FCE decreased the recruitment of inflammatory cell induced by fracture.

\section{Discussion}

FCE is extracted from the freshwater clam (Corbicula fluminea) in Taiwan. The nearest compositions of $100 \mathrm{~g}$ of powdered FCE include $53.4 \mathrm{~g}$ protein, $30.4 \mathrm{~g}$ carbohydrate, $1.1 \mathrm{~g}$ moisture, $11.0 \mathrm{~g}$ crude fat and $4.1 \mathrm{~g}$ ash. FCE contains many bioactivities, including antihypertension, hepatoprotection, antihypercholesterolemia, and antineoplasm (8). Lin et al (11) found that $C$. fluminea crude extracts have the ability to suppress LPS-induced release of pro-inflammatory cytokines by increasing $\mathrm{I} \kappa \mathrm{B}$ protein expression. In the present study it was observed that FCE may effectively reduce tachycardia that is predominantly caused by pain, fever and the release of proinflammatory cytokines following fracture in rats. Instead of analyzing throughout the precise quantities of active gradients of FCE, we examined its anti-inflammatory effect with monocyte-like THP-1 cells and LPS $(1 \mu \mathrm{g} / \mathrm{ml})$, as a stimulator of inflammation. A prior study suggested that clam chloroform water extract had the reverse effect on LPS-induced inflammation via an in vitro method (9); therefore, the present study was designed to elucidate whether it had a consistent anti-inflammatory effect in an in vivo study.

Previous studies have investigated the medication influence on the callus formation and remodeling stages of bone fracture $(13,14)$, while the influence of FCE on the acute stage of fracture is seldom studied. People who suffer from traumatic fracture are often admitted for further surgery, medical care of soft tissue, and administration of analgesics (1). Asians often choose to receive traditional medicine therapy over general medical management under acute trauma condition, such as Chinese herbs or acupuncture $(15,16)$. As such, FCE 

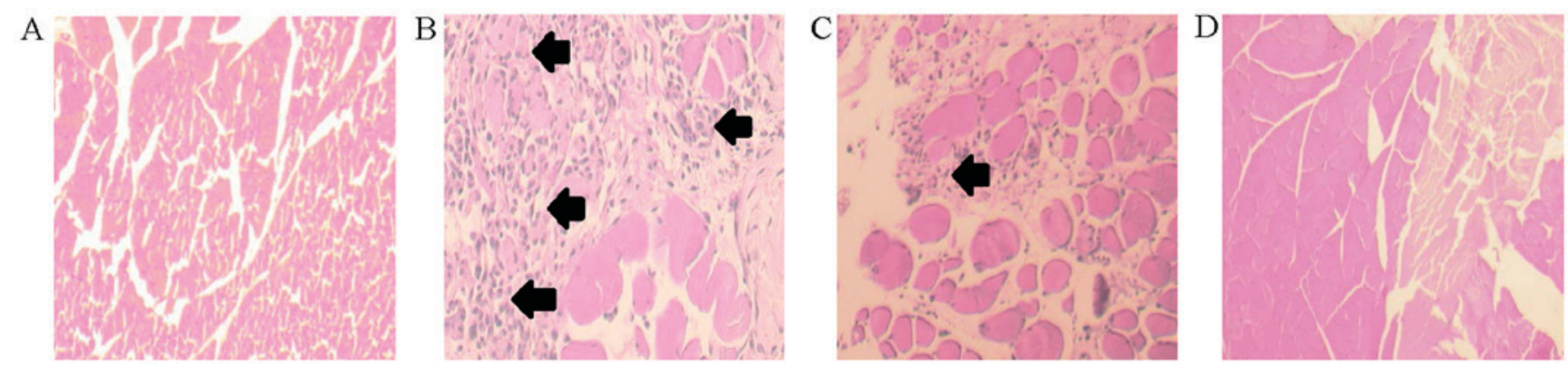

Figure 5. Inflammatory cells infiltration condition of (A) sham, (B) Fx, (C) Fx+FC and (D) FC groups. Histological examination following staining with hematoxylin and eosin (magnification, $\mathrm{x} 400$ ). Black arrows indicate inflammatory cell infiltration.

is a type of Asian-accepted Chinese medicine that may be preferred. The goal of the additional treatment rather than western medicine is to improve the life quality of the patient at the acute stage, including vital signs stabilization, soft tissue swelling faster subsidence and smooth wound healing (16). In previous studies, FCE appeared to effectively suppress the release of pro-inflammatory TNF- $\alpha$ after hemorrhagic shock and protect the liver function of the experimental animals $(17,18)$. The characteristic of anti-inflammatory and liver protection may benefit acute stage of traumatic fracture. Establishment of a conscious rat model $(9,17)$ to observe the effect of FCE on the acute stage of a fracture can simulate our recording vital signs two or three times a day for closely observation the influence of medical therapy on the acute stage. We recorded vital signs of the rats to monitor their instant conditions during the experimental period. The change of HR may show pain condition and CT may show the severity of inflammation. The present results indicate that FCE effectively stabilized the HR of the rats. The rats using FCE also revealed more stable and normal CT compared to those treated with normal saline. The results further suggest that FCE improved the inflammatory condition, as assessed by pro-inflammatory cytokines, and relieved pain, as observed via vital signs, caused by fracturing. This suggests that FCE may be applied as adjuvant therapy for medical treatment at the acute stage of traumatic fracture.

In the present study we can see the fractured rats treated with FCE exhibited lower and more stable levels of CPK, LDH, TNF- $\alpha$, IL-6 and IL-10 level during the 24-h period following fracture. Fracture may be caused by a high energy contusion and result in injury to the surrounding tissue. Both events may cause local soft tissue damage and systemic inflammatory response. Adequate inflammation condition is critical for cascades of bone healing (19), but hyperinflammatory status may exacerbate local tissue damage and vital organ injury (5). The major proinflammatory cytokines associated with response to trauma are TNF- $\alpha$ and IL-6. These are predominantly produced by monocytes and macrophages and cause a variety of frequently additive effects on the inflammatory process at the acute stage (2). TNF- $\alpha$ is an early regulator of the immune response and can induce the release of secondary cytokines, such as IL-6 (2). IL-10 has both proinflammatory and anti-inflammatory effects. The anti-inflammatory effect of IL-10 can reduce the synthesis of proinflammatory mediators at the later period of the acute stage of trauma (4). From this result we know that FCE may be able to relieve the inflammation and soft tissue injury of the acute stage of fracture.
Decrease lymphocyte infiltration and muscle damage and hemorrhage were also noted in our Fx+FC group. Adminssistration of analgesics can have the same effect but may also damage the liver and the kidney, which have been injured by acute trauma condition $(20,21)$. This is the first study to our knowledge that is aimed at intervention at the acute stage of traumatic fracture and application of FCE in this condition. On the basis of the results of this study, we hypothesize that FCE can stabilize the vital signs, improve systemic inflammation and local soft tissue damage condition. These results suggest that FCE may decrease fracture-induced inflammatory reactions and have beneficial regulatory effect on post inflammatory response. Future clinical trials are considered to be done to investigate the effect of FCE on chronic phase of fracture healing.

\section{Acknowledgements}

This study was supported in part by the Hualien Tzu Chi Hospital, Buddhist Tzu Chi Medical Foundation (grant no. TCRD102-57).

\section{References}

1. Giannoudis PV: Current concepts of the inflammatory response after major trauma: An update. Injury 34: 397-404, 2003.

2. HildebrandF, Pape HC and Krettek C: The importance of cytokines in the posttraumatic inflammatory reaction. Unfallchirurg 108: 793-794, 796-803, 2005 (In German).

3. Pape HC, Marcucio R, Humphrey C, Colnot C, Knobe M and Harvey EJ: Trauma-induced inflammation and fracture healing. J Orthop Trauma 24: 522-525, 2010.

4. Stensballe J, Christiansen M, Tønnesen E, Espersen K, Lippert FK and Rasmussen LS: The early IL- 6 and IL-10 response in trauma is correlated with injury severity and mortality. Acta Anaesthesiol Scand 53: 515-521, 2009.

5. Kobbe P, Vodovotz Y, Kaczorowski DJ, Billiar TR and Pape HC: The role of fracture-associated soft tissue injury in the induction of systemic inflammation and remote organ dysfunction after bilateral femur fracture. J Orthop Trauma 22: 385-390, 2008.

6. Kobbe P, Vodovotz Y, Kaczorowski DJ, Mollen KP, Billiar TR and Pape HC: Patterns of cytokine release and evolution of remote organ dysfunction after bilateral femur fracture. Shock 30: 43-47, 2008.

7. Glass GE, Chan JK, Freidin A, Feldmann M, Horwood NJ and Nanchahal J: TNF-alpha promotes fracture repair by augmenting the recruitment and differentiation of muscle-derived stromal cells. Proc Natl Acad Sci USA 108: 1585-1590, 2011.

8. Huang YT, Huang YH, Hour TC, Pan BS, Liu YC and Pan MH: Apoptosis-inducing active components from Corbicula fluminea through activation of caspase-2 and production of reactive oxygen species in human leukemia HL-60 cells. Food Chem Toxico 44: 1261-1272, 2006.

9. Lee RP, Subeq YM, Lee CJ, Hsu BG and Peng TC: Freshwater clam extract decreased hemorrhagic shock-induced liver injury by attenuating TNF- $\alpha$ production. Biol Res Nurs 14: 286-293, 2012. 
10. Hsu CL, Hsu CC and Yen GC: Hepatoprotection by freshwater clam extract against CCl4-induced hepatic damage in rats. Am J Chin Med 38: 881-894, 2010.

11. Lin CM, Lin YL, Tsai NM, Wu HY, Ho SY, Chen CH, Liu YK, Chiu YH, Ho LP, Lee RP and Liao KW: Inhibitory effects of chloroform extracts derived from Corbicula fluminea on the release of pro-inflammatory cytokines. J Agric Food Chem 60: 4076-4082, 2012.

12. An Y, Friedman RJ, Parent T and Draughn RA: Production of a standard closed fracture in the rat tibia. J Orthop Trauma 8: 111-115, 1994.

13. Chrastil J, Sampson C, Jones KB and Higgins TF: Postoperative opioid administration inhibits bone healing in an animal model. Clin Orthop Relat Res 471: 4076-4081, 2013.

14. Savaridas T, Wallace RJ, Muir AY, Salter DM and Simpson AH: The development of a novel model of direct fracture healing in the rat. Bone Joint Res 1: 289-296, 2012.

15. Wong V, Cheuk DK, Lee S and Chu V: Acupuncture for acute management and rehabilitation of traumatic brain injury. Cochrane Database Syst Rev 3: CD007700, 2013.

16. Kan'o T, Han JY, Nakahara K, Konno S, Shibata M, Kitahara T and Soma K: Yokukansan improves distress of medical staff, and cognitive function and motivation in patients with destructive and aggressive behaviors after traumatic brain injury. Acute Med Surg 1: 88-93, 2014
17. Huang KC, Wu WT, Yang FL, Chiu YH, Peng TC, Hsu BG, Liao KW and Lee RP: Effects of freshwater clam extract supplementation on time to exhaustion, muscle damage, pro/anti-inflammatory cytokines, and liver injury in rats after exhaustive exercise. Molecules 18: 3825-3838, 2013.

18. Hua KF, Chen GM, Ho CL, Chen YL, Chen WJ, Huang JF, Perng YS and Lin CC: Freshwater clam extract inhibits inflammatory responses in LPS-activated macrophages by reducing the activation of mitogen-activated protein kinases and NF-kappaB. Nat Prod Commun 7: 1435-1440, 2012.

19. Schmidt-Bleek K, Schell H, Schulz N, Hoff P, Perka C, Buttgereit F, Volk HD, Lienau J and Duda GN: Inflammatory phase of bone healing initiates the regenerative healing cascade. Cell Tissue Res 347: 567-573, 2012.

20. Ungprasert P, Cheungpasitporn W, Crowson CS and Matteson EL: Individual non-steroidal anti-inflammatory drugs and risk of acute kidney injury: A systematic review and meta-analysis of observational studies. Eur J Intern Med 26: 285-291, 2015.

21. Antoine DJ, Dear JW, Lewis PS, Platt V, Coyle J, Masson M, Thanacoody RH, Gray AJ, Webb DJ, Moggs JG, et al: Mechanistic biomarkers provide early and sensitive detection of acetaminophen-induced acute liver injury at first presentation to hospital. Hepatology 58: 777-787, 2013. 